\title{
Radhi Daghfous \\ Les chrétiens de Najrane et leurs rapports avec les musulmans pendant la période fondatrice de l'Islam
}

Située sur la route des caravanes reliant le Yemen aux portes de la Méditerranée, la ville de Najrane était riche grâce à son industrie florissante de tissus et de peaux. Le nom de cette ville est étroitement lié à l'épisode de la persécution des chrétiens par le roi himyarite juif du Yemen Yousouf Dhou Nouwas. Cet évènement survenu au cours du début du VIème siècle ap. JC a été perpétué dans la mémoire des musulmans par le Coran dans la sourate dite des Bourouj (4-85: Ashab al-'Okhdoud).

Il importe d'étudier l'identité des chrétiens de Najrane en précisant leur appartenance tribale et les principaux clans auxquels ils appartiennent ainsi que la question générale de l'entrée du christianisme au Yemen et plus précisément dans la ville de Najrane. Nous analyserons par la suite les rapports entre cette minorité chrétienne arabe avec les musulmans au moment de la période fondatrice de l'Islam c'est à dire à l'époque du Prophète Mohammad et sous les califes Orthodoxes.

\section{1) La situation au Yemen au début du VIème siècle ap JC :}

Avant de devenir une province de l'Etat musulman fondé par Mohammad à Médine en 622 de l'ère chrétienne, le Yemen était placé dans l'orbite persane pendant un demi siècle (578-628) en tant que satrapie après avoir subi l'occupation éthiopienne entre 525 et 575 de l'ère chrétienne.

Au niveau religieux il faut rappeler que le polythéisme fondé sur l'adoration des astres et du soleil était la religion officielle des Sabéens et des Minéens avant d'évoluer progressivement au cours du II è siècle ap .JC vers une forme d'énothérisme qui se caractérise par la domination d'une divinité donnée au dépens des autres divinités dans chaque peuple. Au cours du VIè s le judaïsme fut imposé par la force dans le royaume himyarite par le roi dénommé Dhou Nouwas connu sous le nom de Youssef. Les chrétiens najranites furent d'après la tradition arabe appelés à choisir entre la 
conversion à la nouvelle religion ou la mort brûlés vifs dans un fossé rempli de feu que le roi fit creuser dans la cité pour exécuter sa menace.

A vrai dire le christianisme a été introduit dans sa forme monophysite à partie de la Syrie. Les Romains de Byzance ont encouragé la propagation de cette doctrine par le biais de leurs alliés abyssins eux mêmes convertis au monophysisme. Leur objectif économique et politique plus que religieux était de pouvoir établir leur hégémonie sur le Yemen et mettre la main par la même occasion sur les voies commerciales contrôlées par les yéménites.

C'est dans ce contexte que l'empereur Constance II (337-361) envoya en Arabie du Sud un missionnaire d'origine indienne appelé Théophile y répandre l'arianisme, doctrine qui faisait du Christ un être de nature divine subordonné au Père. Cet émissaire semble avoir eu un succès momentané dans le pays étant donné que sa mission qu'on situe vers 356 de l'ère chrétienne aboutit à la construction de plusieurs cathédrales dans les villes de 'Aden, Dhafar et Najrane.

S'appuyant sur un inventaire de sources épigraphiques et archéologiques variées (inscriptions sud-arabiques et éthiopiennes, graffitis grecs et représentations de croix) J. Beaucamp et Ch. Robin ont pu établir que le christianisme s'est implanté à Najrane dans le courant du Vè siècle, si bien qu'au VIè siècle il y occupait une place prépondérante de sorte que la ville de Najrane apparaît dans les textes comme l'un des centres majeurs du christianisme arabe avant l'Islam à l'égal de Hira capitale de la dynastie lakhmide en Basse Mésopotamie.

J. Ryckmans affirme pour sa part que le christianisme avait pu commencer son véritable essor à Najrane vers le milieu du Vè siècle. Les sources arabes classiques (Ibn Hisham — Tabari — Ibn al Athir) n'ont pas manqué de rapporter des récits souvent légendaires sur cette introduction du christianisme dans la région de Najrane. Il est question notamment des légendes attribuées à Faymiyun et à 'Abdallah al Thamir que A. Moberg a rattachées au cycle perse de Yazdi- Péthion et aux récits de la persécution des chrétiens de Najrane.

Cependant cette première percée de la religion chrétienne fut suivie d'un essor important du judaïsme. Rappelons que celui-ci était présent au nord de l'Arabie et au Yemen bien avant le IVè siècle ap JC. La tradition arabe, notamment Wahb b. Mounabbih, attribue la diffusion du judaïsme au Yemen au roi himyarite As'ad al Kamil surnommé Toubba' al Asgar qui, converti à Yathrib par deux rabbins, imposa la nouvelle religion à ses contribules dans tout le royaume himyarite. Le chroniqueur Ibn Qoutayba va plus loin en signalant que ce souverain réalisa une alliance groupant les tribus 'adnanides de Rabi'a aux tribus yéménites.

Bref tout se passe comme si les rois himyarites du Yemen soucieux de contrer la religion monophysite de leurs adversaires byzantins et abyssins, avaient encouragé la diffusion du judaïsme dans leur propre royaume.

Donc aux Vème et VIème siècles de l'ère chrétienne coexistaient en Arabie le judaïsme (au Yemen et au Hijaz, essentiellement dans les oasis 
de Yathrib, Tayma', Fadak et Khaybar), le christianisme dans sa forme monophysite à Najrane en plus du polythéisme ainsi que diverses sortes de paganisme.

Le règne de Dhou Nouwas, le dernier descendant de la lignée des souverains himyarites au VIème siècle devait porter le conflit entre chrétiens d'une part et juifs de l'autre et par conséquent entre partisans des uns et des autres à son point culminant. La tradition arabe qui remonte à Wahb et qui a été reprise par divers autres auteurs comme Ibn Hisham, Ibn Habib,Tabari, Mas'oudi etc.. fait de dhou Nouwas- sobriquet qui lui a été donné à cause des bouclettes qu'il portait et dont le syriaque masrouq (bouclé) est un calque, de son vrai nom Zor'a b. Toubba' al Asghar b. Hassan b. Toubba' b. Abi Karib, un beau jeune homme qui s'empara du pouvoir en échappant aux entreprises homosexuelles de son prédécesseur, un certain Lakhnia'a Yanouf dhou-alShanatir, en tuant celui-ci et en sauvant du même coup les jeunes hommes yéménites du tribut érotique qu'ils lui payaient.M. Rodinson fait remarquer que le surnom de Yath'ar (vengeur) trouvé sur une inscription portant son nom est peut être une proclamation.

En somme le judaïsme connut un triomphe officiel entre la fin du règne d'Abou Karib (440 ap J.C) et celle du règne de dhou Nouwas (525 de l'ère chrétienne). A cette date dhou Nouwas entreprit - comme il a été mentionné plus haut - une action d'extermination des chrétiens de Najrane qui avaient refusé d'abjurer leur foi chrétienne pour professer le judaïsme, action que les musulmans n'hésitent pas à lier à l'histoire de la tranchée ou de la "fosse embrasée" rapportée par une sourate du Coran (ashab al'okhdoud). Il faut préciser à cet égard que le terme 'okhdoud est un toponyme que Hamdani applique à la ville de Najrane.

Ibn Khaldoun cite une tradition de Hisham Ibn al Kalbi reprise par Nashwane al Himyari d'après laquelle l'action de dhou Nouwas contre les chrétiens de Najrane fut la suite logique à l'appel lancé au roi du Yemen par un juif de la ville dont deux de ses enfants venaient d'être tués par les chrétiens najranites. Pour sa part Hilal al 'Askari relate les évènements comme suit: un juif appelé Daws s'adressa à dhou Nouwas après que deux de ses enfants eurent été tués par les najranites. La cause réside dans le fait qu'ils ont puisé plus que la quantité d'eau nécessaire à l'irrigation de leur champ. Si Nashwane tente de justifier la persécution des chrétiens comme une action de protection du judaïsme contre le christianisme devenu dominant à Najrane; al'Askari impute l'assassinat des deux enfants précédemment cités par la jalousie que les chrétiens vouaient aux juifs à cause de leur richesse. Il est à remarquer que cette tradition est la seule que nous possédons dans les sources arabes qui fasse état d'une rivalité d'ordre économique entre les chrétiens et certains juifs de Najrane.

La fin dramatique de dhou Nouwas sonna la glas de la dynastie himyarite au pouvoir depuis 115 avant JC. Le royaume yéménite perdit définitivement son indépendance politique au profit des Ethiopiens. 
Lintervention éthiopienne dictée essentiellement par des considérations commerciales - à savoir la volonté de protéger la route de la mer Rouge reliant Byzance aux Indes en passant par l'Océan Indien sous une apparence religieuse - venir au secours des chrétiens de Najrane persécutés par dhou Nouwas - aboutit en fait- comme nous venons de le signaler plus haut à une occupation pure et simple du royaume yéménite de 525 à 575 ap J.C. D'autre part il y a lieu de penser que l'invasion éthiopienne était un épisode de la lutte sournoise que se livraient au Vlème siècle les deux grands puissances de l'époque, en l'occurrence Byzance et la Perse, et leurs alliés respectifs (éthiopiens et himyarites), lutte dont l'enjeu n'était rien de moins que l'établissement de leur propre hégémonie tant politique qu'économique sur la totalité de la Péninsule Arabique.

Les éthiopiens profitèrent de leur domination soutenue par Byzance pour répandre le christianisme dans le royaume en construisant des églises notamment la cathédrale d'al Qalis à San'a et en procédant à expurger le judaïsme du Yemen.

Abraha qui évinça son rival Ariyat avant de prendre le pouvoir au Yemen est désigné dans les sources arabes comme l'auteur de l'expédition contre la Mecque en 570 ap JC . A ce propos Jawad 'Ali fait remarquer que la cause essentielle de cette expédition contre la Ka'ba ne pouvait être la profanation d'al Qalis par des éléments arabes issus de Kinana et de Kha'am que les auteurs arabes ont invoquée. Il pense plutôt que le souverain éthiopien voulait, par cette action unifier sous la bannière chrétienne, l'Arabie occidentale et méridionale et permettre aux Byzantins, ses alliés politiques et religieux, d'échapper aux Perses qui, maitres des routes commerciales menant aux Indes, imposaient des taxes très élevées sur les marchandises importées d'Extrême Orient (Ceylan - Inde). D'autre part il se demande si Byzance n'avait pas encouragé 'Abraha pour conquérir la Mecque et fonder ainsi un "front chrétien" dirigés contre les Perses et leurs alliés arabes les Lakhmides de Hira.

Mais l'échec de l'expédition d'Abraha contre la Mecque aboutit quelques années plus tard et plus précisément en 575 à l'intervention persane pour appuyer Sayf b. dhi Yazin - un notable yéménite appartenant à la tribu de Himyar qui s'était converti au judaïsme d'après Nashwan al Himyari — dans sa lutte contre la présence éthiopienne. Finalement les éthiopiens furent chassés du Yemen au profit d'abord de Sayf — intronisé comme roi avant d'être à son tour assassiné en 578 - ouvrant ainsi la porte à une occupation du pays par les perses qui en firent une satrapie dans le cadre de leur vaste empire sassanide et ce jusqu'en 628 (date de conversion à l'Islam du dernier satrape Badhan).

\section{2) La politique de Mohammad au Yemen au début du VIIème è siècle:}

Au début du VIIème siècle le Yemen était - comme nous venons de le préciser - une satrapie sassanide dirigée par un satrape nommé 
directement par l'empereur perse établi à Ctésiphon. Il n'est pas question d'étudier en détails la période de l'occupation perse du Yemen qui a duré un demi siècle (578-628). Notre but est plutôt l'analyse de la politique de Mohammad au Yemen d'une façon générale et à Najrane plus particulièrement pour comprendre les rapports établis entre les chrétiens najranites et les musulmans.

Après la fondation de l'Etat musulman à Médine aux lendemains de la hijra en 622 le Prophète Mohammad entreprit une série d'actions en Arabie dans le but évident de convertir les tribus arabes à l'Islam et de procéder à l'unification de l'ensemble de la Péninsule sous son autorité. Il usa à la fois de la violence et de la diplomatie sans oublier les moyens économiques qu'il n'a pas hésité à mettre à contribution pour réaliser son projet.

Après la conquête de la Mecque en l'an 8 de l'Hégire suivie de la soumission des qourayshites au pouvoir médinois les tribus arabes de l'ensemble de la Péninsule envoyèrent des délégations à Médine pour présenter leur bay'a au Prophète et officialiser leur entrée à l'Islam (c'est l'année dite des woufoud - année des députations - qui coincide avec l'an 9 de l'Hégire).

En ce qui concerne le Yemen et les tribus yéménites il est intéressant d'observer que Mohammad usa à leur égard de diplomatie et de moyens économiques et il ne dut employer la violence et la force qu'en de rares occasions.

Rappelons tout d'abord que Mohammad ne se serait pas contenté pour le Yemen de correspondre avec la gouverneur perse établi à San'a, en l'occurrence Badhan ou Badham, mais il a pris soin de contacter un certain nombre de notables locaux et de princes appelés 'adhwa' et 'abahila. Ceci semble indiquer qu'il était non seulement bien informé de l'émiettement de l'autorité politique et du démembrement territorial du Yemen au début du VIIème siècle mais qu'il avait aussi sa propre stratégie qui différait de celle suivie avec les tribus du nord et de l'est de la Péninsule.

Nous avons dénombré deux missions confiées par Mohammad respectivement à Khalid b. Al Walid et 'Ali b. Abi Talib auprès des gens du Najrane. L'expédition de Khalid auprès des Bal Harith b. Ka‘b (un batn de la grande confédération tribale des Madhij) eut lieu en rabi' Ier ou joumada Ier de l'an 10 alors que celle d'Ali dans le territoire des Madhij et à Najrane se passa au mois de ramadhane an 10 de l'hégire.

D'autre part il faut préciser que l'action de Mohammad en direction des tribus au sud de la Mecque avait touché tous les habitants de Najrane, à la fois les chrétiens et les païens.

Mais nous allons porter notre attention sur les rapports avec les chrétiens du Najrane. Au départ Mohammad dut adresser aux évêques de la ville une lettre que Hamidallah situe entre l'an 8 et l'an 9 et plus précisément avant l'expédition de Tabouk (rajab an 9/ octobre 630) et avant la conclusion du traité de paix de Najrane.

Voici le texte de cette lettre : 
"De Mohammad Envoyé de Dieu aux évêques de Najrane: Par le nom de Dieu d'Abraham, d'Isaac et de Jacob! Or donc je vous appelle hors de l'adoration des créatures à l'adoration de Dieu. Si donc vous refusez, ce sera la jizya ; si vous refusez aussi la jizya, je vous déclarerai la guerre. Salutations!".

Il s'agit d'un véritable ultimatum qui leur donnait à choisir entre la conversion à l'Islam, la soumission et le paiement - à titre de dhimmis ou protégés - de la capitation (jizya) ou la guerre. Les najranites dépêchèrent aussitôt à Médine une députation forte de 60 membres dont 14 notables de la ville. Dirigée par trois chefs: l'évêque proprement dit (al Harith b. 'Alqama) issu de la tribu de Bakr b.Wa'il et plus précisément du groupement des Banou Rabi'a; le vicaire ou lieutenant (al-'Aqib 'Abd al-Masih) issu de la tribu de Kinda et enfin le chef de la caravane (al-sayyid al-'Ayham Shourahbil) originaire de la tribu des Bal Harith b. Ka'b affiliée aux Madhij. Les médinois furent - nous dit-on - impressionnés par les habits et les chameaux de ces najranites venus voir le Prophète Mohammad. Après de longues discussions sur les matières religieuses et surtout la position de l'Islam concernant Jésus, les dirigeants de Najrane décidèrent de conclure une paix, moyennant soumission politique et paiement d'une taxe.

Un document de paix (soulh) fut rédigé entre les deux parties. Ce traité garantissait notamment au clergé de Najrane ses intérêts et assurait une liberté de conscience et de culte aux najranites ainsi qu'une garantie contre toute atteinte à leurs personnes et leurs biens. En échange les chrétiens de Najrane s'engageaient à payer annuellement aux musulmans un tribut de 2000 vêtements d'une valeur d'une once, 1000 en rajab et 1000 en safar ; à prêter au gouvernement de Médine des armes (30 cottes de maille) et des bêtes de transport (30 chevaux, 30 chameaux) en cas de troubles dans le Yemen et enfin à renoncer à l'usure.

Voici le texte de ce traité :

"Au nom de Dieu, le Clément, le Miséricordieux. Voici ce qu’a fixé le Prophète Mohammad pour les habitants de Najrane sur qui il avait autorité, au sujet de toute (récolte) de fruits, de toute (monnaie) jaune ou blanche, de tout esclave. Or il leur fut généreux en leur abandonnant le tout contre 2000 habits d'une once, dont 1000 à remettre chaque mois de rajab et 1000 à chaque mois de safar, avec (en surcroît) une once d'argent par habit (autre lecture: habits équivalent chacun à une once d'argent), étant entendu que tout excédent sur l'impôt ou tout manque à la quantité d'onces serait mis en compte; que tout qu'ils remettraient, en outre, comme cottes de mailles ou comme chevaux ou montures (chameaux) ou autres objets, leur serait mis en compte; qu'aux najranites incomberait le soin de l'approvisionnement et les fournitures de mes envoyés pour la durée maximum d'un mois (autre lecture: 20 jours) ; qu'au cas d'une guerre ou d'un crime dans le Yemen, ils (les najranites) auraient à prêter à mes (envoyés) 30 cottes de mailles, 30 chevaux et 30 chameaux; que ce qui aurait péri des cottes de mailles; chevaux, montures et autres objets prêtés à mes envoyés, resterait à la charge de nos envoyés jusqu'à sa restitution aux najranites. 
La protection de Dieu et la garantie du Prophète Mohammad, Envoyé de Dieu, s'étendent sur Najrane et alentours, soit sur leurs biens, leurs personnes, la pratique leur culte, leurs absents et présents, leurs familles, leurs sanctuaires et tout ce qui, grand ou petit, se trouve en leur possession. Aucun évêque ne sera déplacé de son siège, épiscopal, ni aucun moine de son monastère, ni aucun prêtre de sa cure. Aucune humiliation ne pèsera sur eux, ni le sang d'aucune vengeance antérieure à la soumission. Ils ne seront ni rassemblés, ni assujettis à la dîme. Aucune troupe ne foulera leur sol. Et lorsque l'un d'eux réclamera un du, l'équité sera mise parmi eux. Ils ne seront ni oppresseurs, ni opprimés. Et quiconque d'entre eux pratiquera à l'avenir l'usure, sera mis hors de ma protection. Aucun homme parmi eux ne sera responsable de la faute d'un autre. Donc, la garantie de Dieu et l'assurance du Prophète Mohammad, Envoyé de Dieu sanctionne le contenu de ce récit, jusqu'au jour où Dieu manifestera Son autorité, tant qu'ils (les najranites) demeureront dans de bonnes dispositions et agiront en conformité avec leurs devoirs sans subir aucun outrage.

Ont témoigné: Abou Sofiyan Ibn Harb, Ghaylan Ibn 'Amr, Malik b.' Awf (du Bani Nasr),'Aqra' b.Habis al Hanzali et Moughira b. Shou'ba. Les présentes ont été écrites par 'Abdallah b.'Abi Bakr".

Ce traité exemptait - comme on vient de le voir - les najranites du paiement de la jizya que les autres dhimmis du Yemen devaient aux musulmans mais il les astreignait par contre à fournir une fidiya, une sorte de forfait annuel évalué à 2000 robes; chaque robe valant une waqiyya ou 40 dirhams; d'où une somme totale de 8000 dirhams ou 800 dinars. Ibn Sallam qualifie les chrétiens de Najrane de "ahl al fidiya" au sens de "mousalaha" ou "mou'ahada", autrement dit des gens avec lesquels un traité de paix est intervenu. En plus de cette fidiya payable en deux moitiés: 1000 au $2^{\text {ème }}$ mois de l'année lunaire (c'est-à-dire safar) et 1000 autres au $7^{\text {ème }}$ mois (c'est-à-dire rajab), les najranites étaient tenus de prêter aux musulmans des armes et des bêtes de transport et d'assurer l'hospitalité (une sorte de droit de gîte) aux agents du Prophète pendant 20 ou 30 jours d'affilée. Le Prophète Mohammad consentit également aux chrétiens de Najrane une série d'avantages comme la liberté de garder leurs évêques et leurs prêtres, l'interdiction pour les musulmans de prélever sur eux les dîmes ('oshour) et de les expulser de leurs terres tant qu'ils s'engagent à renoncer à l'usure ( $a$ l riba') et à ne pas trahir les musulmans.

Ce traité revêt sans aucun doute une grande importance dans la mesure où il donne une orientation constante à la politique islamique envers les non-musulmans. Pourtant le Prophète Mohammad avait déjà entrepris une telle politique à l'égard des juifs aussi bien au Bahrayn qu'à Khaybar et à Tabouk. Mais le traité de Najrane lui donna un développement définitif en l'étendant aux chrétiens de 'Ayla, 'Adhrouh et 'Adhri'at. Ce qui importe le plus de retenir c'est que ce traité assure aux chrétiens de Najrane des privilèges que ni les chrétiens des Banou Taglib ni ceux de 'Ayla n'avaient réussi à obtenir. 
Les sources musulmanes citent une autres lettre adressée par le Prophète Mohammad aux chrétiens de Najrane et à leur évêque, lettre dans laquelle, en échange de leur loyauté à l'égard des musulmans, les najranites sont assurés de la garantie de Dieu et de Son Envoyé pour leurs personnes, leurs biens et leurs offices.

Voici le texte de cette lettre:

"Au nom de Dieu, le Clément, le Miséricordieux. Du Prophète Mohammad à l'évêque 'Abou'l Harith, aux évêques de Najrane... à leurs prêtres et à ceux qui les suivent, ainsi qu'à leurs moines. A eux, reviennent tout ce qui se trouve dans leurs main,s, que ce soit peu ou beaucoup, leurs églises,leurs oratoires et leurs monastères, à eux également la protection de Dieu et de Son Envoyé. Aucun évêque ne sera déplacé de son siège épiscopal, ni aucun maître de son monastère, ni aucun prêtre de sa cure. Aucun de leurs droits, ni de leurs pouvoirs ne sera changé, non plus aucune chose (coutume?) à laquelle ils sont habitués. Sur cela, la protection de Dieu et de Son Envoyé est assurée pour toujours, tant qu'ils se comporteront sincèrement et agiront en conformité avec leurs devoirs. Ils ne seront ni assujettis à l'oppression, ni eux-mêmes oppresseurs . Ecrit par Al Moughira".

En somme la politique mohammadienne envers les chrétiens du sud, d'une façon générale, et plus particulièrement envers ceux de Najrane, fut d'après M. Watt la même que celle suivie au nord de Médine et de la Mecque, bien que leur attitude, en tant que chrétiens nestoriens peu attachés à l'empire perse pourtant hostile au monophysisme mais ayant des liens politiques avec les juifs du Yemen, ait été différente de celle de leurs corréligionnaires du nord face à Byzance. Quoiqu'il en soit les chrétiens de Najrane sont devenus, à la faveur du traité de paix conclu avec le Prophète, des alliés des musulmans, entrant ainsi dans la sphère de ce que M. Watt appelle "la Pax Islamica" ou système de sécurité médinois que M.A. Shaban qualifie pour sa part de "Commonwealth de Médine".

Comment les choses ont-elles évolué après la mort du Prophète?

\section{3) Les rapports entre les Musulmans et les chrétiens de Najrane après la mort du Prophète Mohammad en 632:}

A vrai dire le traité de Najrane a été renouvelé par le successeur de Mohammad à la tête de l'Etat médinois à savoir le calife Abou Bakr dans les termes que voici:

“Au nom de Dieu, le Clément, le Miséricordieux. Voici ce qu'a fixé par écrit le serviteur de Dieu Abou Bakr, successeur du Prophète Mohammad Dieu prie pour lui et salue — pour les habitants de Najrane :

Il les garantit par la garantie de Dieu et l'assurance du Prophète Mohammad Envoyé d Dieu — Dieu prie pour lui et salue — garantie allant à leurs personnes, à leurs terres, à la pratique de leur culte, à leurs biens,à leur personnel, à leurs fantassins, à leurs absents et présents, à leurs évêques, 
à leurs moines, à leurs églises, à toute chose grande ou petite qui se trouve en leur possession. Qu'ils ne puissent être rassembles, ni assujettis à la dîme ;qu'aucun évêque ne soit déplacé de son siège épiscopal, ni aucun moine de son monastère (tout cela) en accomplissement de ce que le Prophète Mohammad leur avait fixé par écrit. Et sur tous les points contenus dans cet écrit, joueront à jamais la garantie de Dieu et la garantie du Prophète Mohammad - la Paix soit sur lui ! Et à eux (c'est-à-dire les najranites) d'avoir de bonnes dispositions et bien faire en ce qui concerne leurs devoirs».

Témoins: Al Moustawrid b. 'Amr de la tribu des Banou-l Qayn 'Amr ou 'Amir le mawla d'Abou b Bakr, Rashid b. Houdhayfa et al Moughira b. Shou'ba lequel a écrit les présentes"

Mais le calife 'Omar - dans le cadre de sa politique visant à nettoyer la Péninsule Arabique de toute présence non musulmane - décida d'expulser les chrétiens de Najrane vers l'Iraq. Le traité conclu auparavant par le Prophète Mohammad et renouvelé par le calife Abou Bakr fut donc remis en question.

Comment expliquer cette mesure d'expulsion?

De l'avis des sources musulmanes, ce sont les najranites eux-mêmes qui, en s'adonnant à la pratique de l'usure, ont failli à leurs engagements contenus dans le traité signé avec Mohammad, suscitant du coup la décision du calife 'Omar de les expulser de l'Arabie. En réalité il ne s'agit là que d'un prétexte. La raison essentielle se trouve à notre avis dans le hadith qu'on attribue au Prophète Mohammad et qui stipule "qu'il ne fallait pas que deux religions puissent coexister en Arabie". Il est intéressant de noter que ce hadith prophétique rapporté par 'Omar b. 'Abd al 'Aziz, est également cité par Motahhir b. Rafi' pour expliquer l'expulsion des juifs de Khaybar et le partage de l'oasis en 16 ou 36 parts. D'autre hadiths attribués au Prophète Mohammad et rapportés par de nombreux traditionnistes (Jabir , Ibn 'Omar, al-'A'mash etc.) vont dans le même sens, c'est-à-dire la justification de l'expulsion des arabes dhimmis (juifs, chrétiens) de la Péninsule Arabique.

Une autre tradition de Waqi ${ }^{\mathrm{b}} \mathrm{b}$. Jarrah que cite Baldhouri présente les choses autrement: ce sont les najranites qui, à la suite de leur accroissement démographique important (on parle de 40000 personnes), se sont trouvés trop à l'étroit et ont demandé au calife 'Omar de les transférer ailleurs. Evidemment il nous est très difficile d'accepter un tel scénario à moins de relier cette tradition à la version de Abou Youssef. Ce dernier fait remarquer que 'Omar avait pris la décision d'expulser les najranites en Iraq parce qu'il craignit à la fois leur force (ils avaient nous dit-on beaucoup d'armes et de chevaux) et leur nombre (il a été question de 40000 personnes) pour les musulmans.

Par conséquent le précédent hadith attribué au Prophète pour justifier l'expulsion des chrétiens de Najrane en dehors de la Péninsule Arabique semble avoir été forgé a posteriori, mais il n'empêche que la mesure du calife 'Omar avait l'avantage de débarrasser les musulmans d'adversaires potentiellement dangereux. 
Trois autres documents mentionnés par les sources musulmanes méritent aussi d'être signalés pour mieux éclairer la situation des chrétiens de Najrane.

Le premier texte est un message (kitab) que le calife 'Omar b. alKhattab avait remis aux najranites avant leur expulsion vers l'Iraq et qui nous a été rapporté par Ibn Sallam d'après une tradition d'Ibn Sirin.

Le voici:

"Au nom de Dieu, le Clément, le Miséricordieux. De 'Omar commandeur des croyants à tous les habitants de Rou'ach .Soyez en paix. Je vous mande les louanges de Dieu hors duquel il n'y a point de dieu. Comme suite à cela, vous avez prétendu être des musulmans, puis vous avez apostasié par la suite. Celui d'entre vous qui manifeste son repentir et revient à la voie droite, il ne lui sera pas tenu rigueur de son apostasie et nous serons pour lui d'une bonne compagnie. Sachez vous souvenir pour ne pas être condamné. Que celui d'entre vous qui se convertit à l'Islam soit félicité. Par contre celui qui tient à sa foi chrétienne, sachez que ma garantie sera retirée d'ici le 20 du mois de ramadhan à tous les chrétiens de Najrane.

Comme suite à cela, Yala prétend se disculper de toute entreprise de contrainte ou de torture exercée sur quiconque pour qu'il se convertisse à l'Islam...

J'ai prescrit à Ya'la de collecter la moitié de ce que vous auriez récolté la terre, et je n'ai nullement l'intention de vous confisquer celle-ci tant que vous demeurez dans la voie droite".

Retenons de ce document important deux idées essentielles: d'abord l'invitation du calife 'Omar aux chrétiens de Rou'ach - un village proche de Najrane - à se convertir à l'Islam avant la date du 20 ramadhan de l'année 13 de l'hégire, sous peine de se voir retirer la qualité de dhimmis jouissant de la protection des musulmans; ensuite l'appel au gouverneur du Yemen, en l'occurrence Ya'la b.'Omayya, à procéder au partage des récoltes des najranites en question, décision qui nous rappelle sans aucun doute une autre mesure prise par le Prophète Mohammad à l'égard des juifs de Khaybar (moushatara ) à l'issue de la conquête de leur oasis en l'an 8 de l'hégire.

Le second document est une lettre adressée par la calife 'Omar au gouverneur du Yemen Ya'la b.'Omayya et dans laquelle il lui ordonnait de prélever sur les terres appartenant aux najranites expulsés vers l'Iraq un impôt foncier (kharaj) dont le taux variait en fonction du procédé d'arrosage du sol et des types de cultures. C'est le qadhi Abou Youssef qui, se référant à une tradition de Yala en personne, précise qu'une sorte de "mouqasama" fut notifiée dans la lettre en question. Ce partage entre les occupants de la terre et le calife porte aussi bien sur les terres plantées d'arbres fruitiers et de dattiers que sur celles cultivées en céréales. Il est notamment spécifié que le produit de la récolte devait être partagé à raison de I/3 pour les occupants du sol et $2 / 3$ pour le calife et les musulmans s'il s'agissait de terres arrosées par la pluie ; par contre si les terres étaient irriguées par les instruments, $2 / 3$ du produit de la récolte allaient aux occupants et $1 / 3$ seulement au calife et au bayt al mal. 
Il est clair d'après ce document que c'est 'Omar en personne qui fixait le taux du kharaj sur les produits de la terre (cultures et fruits) en prenant en considération - à l'instar des terres à dimes - les types d'arrosage du sol. Remarquons au passage que le terme de kharaj - au sens de taxe foncière - est utilisée pour la première fois au Yemen qui est classé par les ouvrages de fiqh parmi les territoires à dîme, ce qui veut dire que les propriétaires fonciers payaient soit le 'oshr soit le vingtième sur leurs terres.

Enfin le dernier document est tout simplement un nouvel acte concédé aussi par le calife 'Omar aux najranites installés en Iraq et plus précisément dans le village de Najraniyya, et dans lequel il les exempte du paiement pendant deux ans - de la capitation (jizya) et leur renouvelle la protection et la garantie de Dieu et des musulmans.

Voici le contenu de cet acte :

"Au nom de Dieu le Clément, le Miséricordieux.

Voici ce qu'a fixé par écrit 'Omar, commandeur des croyants pour les habitants de Najrane. Quiconque d'entre eux se déplacera sera sauf de par la sauvegarde de Dieu. Aucun musulman ne lui fera tort, tout cela en accomplissement de ce que leur fixèrent par écrit le Prophète Mohammad et Abou Bakr.

Ajouter que ceux d'entre les gouverneurs d'Iraq chez qui ils se rendront, leur permettrait de cultiver la terre .Et tout ce qu'ils y auront travaillé leur ira en aumône et cela en remplacement de la terre, nul n'ayant la faculté ni le droit de le leur contester.

Ajouter encore : que tout musulman qui se rendra auprès d'eux comme gouverneur devra leur prêter aide contre leurs oppresseurs, car ils font partie des gens à protéger. Ils seront dispensés de la jizya durant 24 mois à compter du jour de leur arrivée et ils n'auront à supporter quoique ce soit que dans la mesure de leur travail; obesrvance et non violation ni transgression. Témoins: 'Othman b.'Affan, Mo'awiyya b.Abi Fatima lequel a écrit les présentes".

Dans son récit des évènements de l'an 13, Tabari corrobore les indications rapportées par Abou Youssef, Ibn Sallam et Baladhouri concernant les conditions d'expulsion en Iraq des chrétiens de Najrane et ajoute que le calife 'Omar avait joint à son agent Ya'la b.'Omayya de leur proposer, avant de quitter leurs maisons et leurs biens, soit de rester fidèles à leur foi et d'accepter la mesure d'expulsion, soit au contraire de se convertir à l'Islam et de se maintenir à Najrane. Il lui avait également demandé de procéder au mesurage des terres des najranites, afin qu'elles soient soumises au kharaj.

Rappelons par ailleurs que les najranites installés en Iraq à Najraniyya reçurent dans la lettre - que le calife 'Omar avait adressée, en sha 'ban an 27 /milieu du mois de mai an 648, à son gouverneur al-Walid b. 'Oqba le renouvellement de la dhimma de Dieu et des musulmans ainsi qu'une réduction de le fidiya (1970 ou 1800 vêtements au lieu de 2000 pièces). Plus tard le calife 'Ali b. Abi Talib devait rédiger pour eux, en joumada 37 un 
nouvel acte qui stipulait notamment le renouvellement de sa protection et la garantie de leurs biens. La fidiya des najranites fut par la suite rabaissée de 200 vêtements par le calife omeyyade Mo'awiyya b. Abi Sofiyan, puis ramenée de nouveau à 1800 pièces par le gouverneur d'Iraq al Hajjaj b. Youssef alTaqafi avant que le calif 'Omar b. Abd al-'Aziz ne la fixe définitivement à 200 pièces par an, d'une valeur totale de 8000 dirhams.

\section{Conclusion:}

Au total les najranites - en tant que dhimmis jouissant de la protection de l'Etat musulman — ont bénéficié de privilèges importants. Cette minorité religieuse dont l'existence remonte au Vème siècle a su garder son identité d'abord en résistant à la persécution du roi himyarite Youssef dhou Nouwas en 524 ap JC, ensuite en négociant avec le Prophète Mohammad un traité de paix qui lui a garanti un certain nombre d'avantages que les autres dhimmis (chrétiens arabes de Bani Taghlib et juifs des oasis du Hijaz notamment) n'ont pu obtenir.

Certes cette communauté najranite fut expulsée d'Arabie par le calife 'Omar mais elle parvint encore une fois à arracher certains avantages qui en disent long sur les rapports étroits tissés avec les musulmans durant l'époque fondatrice de l'Islam. 


\section{Bibliographie}

\section{Les sources:}

'Abid b. Shariiya al Jourhoumi. 'Akhbar 'Abid b. Shariyya 'an bilad al Yaman. San'a, 1347. Al 'Askari (Abou Hilal). Kitab al 'awa'il. Beyrouth, 1987.

Ibn Al Athir. Al kamil fi'l tarikh. I. Beyrouth, 1967.

Baladhouri. Foutouh al bouldan. Beyrouth, 1952.

Dinawari. Al-akhbar al-tiwal. Le Caire,1960.

Diyar Bakri. Tarikh al-khamis fi ahwal anfas nafis. Le Caire, 1866-I867.

Hamdani. Al Iklil. II. Baghdad, 1980.

Ibn Habib. Kitab al mouhabbar, Beyrouth, s. d.

Ibn Hisham. Sirat al-nabi. I. Le Caire, 1955.

Ibn al-Kalbi (Hisham). Kitab al-Asnam, Le Caire,1965.

Ibn Mounabbih (Wahb). Kitab al-tijan fi moulouk Himyar. San'a, 1347.

Mas'oudi. Mourouj ad-dhahab wa ma'adin al-jawhar. I. Beyrouth, 1962.

Nashwan al-Himyari. Kitab moulouk Himyar wa aqiyal al Yaman. Le Caire, 1958-1959.

Ibn Qutayba. Al-ma'arif. Le Caire, 1960.

Ibn Sallam. Kitab al-Amwal. Le Caire, 1965.

Al Souhayli. Al-rawdh al-ounouf, I. Le Caire, 1914.

Tabari. Tarikh al rousol wa'l moulouk. II. Le Caire,1969.

Ya'qoubi. Tarikh. I. Beyrouth, 1970.

Abou Youssef. Kitab al Kharaj. Le Caire, n.d.

\section{Les Travaux:}

Al-Akwa' Al-Qadhi Mohammad. Al-watha'iq as-siyassiya al-yamaniyya min qoubayl al-Islam ila sanat 332 h. Baghdad, 1976.

Al'Ali Salah Ahmad. Tarikh al'arab al qadim wa'l bi'tha. Beyrouth, s.d.

Beeston A.F.L. Judaisme and christianity in pre-Islamic Yaman // Chelhod J. L'Arabie du Sud. T. III. P., 1985. P. 271-278.

Daghfous $R$. Le Yaman islamique des origines jusqu'à l'avènement des dynasties autonomes, I. Tunis, 1995.

Le régime fiscal et l'organisation financière au Yemen à l'époque des Woulat // Cahiers de Tunisie. 1990, n 151-154. P. 65-112.

Mohammad wa nash'at al Islam, L'or du temps. Tunis, 2005.

Jawad 'Ali. Al-moufassal fi tarikh ak 'arb qabla al-Islam, II. Baghdad, 1968. 
Encyclopédie de l'islam, Nouvelle édition. Leiden, 1960 et sq.

Hamidoullah M. Majmou'at al-watha'iq al-siyassiya li'l 'ahd al nabawi wa'l khilafa arrashida. Le Caire, 1956.

Pigulevskaya N.U. Les rapports sociaux à Najrane au début du VIè s de l'ère chrétienne // Journal of the Economic and Social History of the Orient. I960, vol. III. P. 113-130; vol. IV, p. 1-14.

Robin C.J., Beaucamp J. Le christianisme dans la péninsule Arabique d'après l'épigraphie et l'archéologie // Travaux et mémoires. Centre de Recherche d'Histoire et de Civilisation de Byzance, Hommage à Paul Lemerle. N 8. P., 1981. P. 45-61.

Ryckmans J. Le christianisme en Arabie du sud préislamique // L'oriento cristiano nelle storia della civilita. Roma, 31 Marzo-3 April 1963; Firenza, 4 April 1963, Accademica nazionale dei Lincei, Anno CCCLXI, 1964, Problemi attuali di scieznza e di culture, qaderno $n^{\circ}$ 62. Roma, 1964. P. 413- 454.

La persécution des chrétiens himyarites au VIème siècle d'après les récentes découvertes en Arabie Centrale, Istanboul, 1966.

Smith S. Events in Arabia in the 6th Century A.D // Bulletin of the School of Oriental and African Studies. 1954, vol. XVI. P. 425-468.

Watt M. Mahomet à Médine. P., 1978. 